\title{
Rechtsgeschichte
}

\section{Eliana Augusti}

\section{Le ragioni dell'saltro<}


te, hat sich von der Überschwangssemantik des `Neuen`, in die er infolge des Weimarer Desasters hineinversetzt wurde, nicht überwältigen lassen. »Die Übersetzung ausländischer Gesetze in die türkische Sprache und ihre Verkündung als türkische Gesetze in den Jahren I 926 ff. war an die Erwartung geknüpft, daß die türkische Bevölkerung ... sich in ihrem täglichen Leben nicht mehr nach dem gewohnten überkommenen islamischen Recht (Scheriat), sondern nach den ganz anderen neuen gesetzlichen Vorschriften richtete, eine Erwartung, von der ich zwar ausging, obwohl sie sich erst nach vielen Jahrzehnten und dann auch nicht vollständig erfüllte. ${ }^{4}$

\section{Le ragioni dell'>altro ${ }^{*}$}

Minorités en Turquie. Turcs en minorité è un testo sicuramente destinato a rivitalizzare il dibattito sul tema del multiculturalismo in Europa. La nuova ondata di migrazioni degli ultimi cinquant'anni, accanto alla graduale messa in discussione del concetto di stato-nazione, ha posto il pensiero giuridico europeo davanti alla necessità di cercare nuove soluzioni al problema della gestione dell'>altro<. Con la crisi dell'ideologia universalistica della modernità, formalmente egualitaria ed emancipatoria, è riemerso ciò che la stessa modernità aveva provato ad occultare. Particolarismi, dimensione comunitaria e differenze sono tornati al centro della riflessione giuridico-politica e sono divenuti i termini di quella società multiculturale che Gerd Baumann ha rappresentato nella complessa relazione stato-nazione/identità culturale/religione.
Unser Autobiograph sieht: Auch die Herausbildung dieser normativen Ordnung blieb von den Ablagerungen der Geschichte nicht einfach unberührt, so entschlossen man auch war, smodern $<$ zu werden. Uns Heutigen, die wir über Rechtstransfer und legal transplants sehr gern sehr theoretisch reflektieren, ${ }^{5}$ könnte der sehr empirische Lebensabschnittsbericht des emigrierten Hochschullehrers und "Kodifikatörs " Anstoß sein, Theorie und Quellen beisammenzuhalten.

\section{Gerd Bender}

Samim Akgönül parte da qui. Combinando approcci differenti che risentono finanche delle suggestioni dei cultural e post-colonial studies, egli affronta la questione della convivenza fra culture diverse all'interno degli ordinamenti giuridici, politici e sociali di Francia, Germania e Grecia da una parte e Turchia dall'altra. La narrazione privilegia la prospettiva comparativa, la sola, a suo giudizio, in grado di individuare le analogie (»le cas unique n'a pas de cause «) e, allo stesso tempo, di scongiurare pericolose assolutizzazioni o »modélisations abusives «. Al centro dello studio, però, Akgönül non pone l'individuo, ma il gruppo inteso come insieme di individui associati volontariamente, gruppo che si interseca costantemente con altri gruppi. Non si tratta di un dialogo oggettivamente liberale, ma di un contraddittorio permanentemente conflittuale tra una maggioranza e una o più mi-

\footnotetext{
4 S. I42. Siehe des Näheren E. E. Hirsch, Rezeption als sozialer Prozeß. Erläutert am Beispiel der Türkei, Berlin I98 г. Zur postosmanischen Modernisierungsgeschichte zählen auch jene minderheitenpolitischen Verwerfungen und Exzesse, welche die Genese des türkischen Nationalstaats begleiteten. Insbesondere

กั die eklatanten Menschenrechts-

nischen Bevölkerung begangen wurden, sorgen bis heute für Zündstoff.

5 Ein kleines Glanzstück der Rechtstheorie: M. T. FöGEN, G. Teubner, Rechtstransfer, in: Rg 7 (2005) $38 \mathrm{ff}$.

\footnotetext{
* SAmim AkgönüL, Minorités en Turquie, Turcs en minorité. Regards croisés sur l'altérité collective dans le contexte turc (Les cahiers du Bosphore 55), Istanbul: Isis 2010, I $46 \mathrm{~S}$., ISBN 978-975-428405-8
} 
noranze. La prospettiva di Akgönül è quella del confine; la dimensione dialettica è quella del dentro/fuori, del noi/gli altri, dell'inclusione/ esclusione, della maggioranza/minoranza. Ciò che si conserva è il continuo rinvio all'`occhio interno dell'identità, maggioritaria o minoritaria che sia. Ma è in condizioni di minoranza che l'identità, e l'identità religiosa in particolare, trova la sua dimensione di forza. Si parla di 'minoranze attive`, di minoranze che reclamano una legittimità d'appartenenza all'insieme della società senza rinunciare a preservare la propria differenza. La minorité, vista come »fait social total «, è un'alterità collettiva della quale è necessario definire l'entità. Il punto d'osservazione è quello turco, nella duplice prospettiva chez les Turcs e pour les Turcs.

Il primo capitolo funge da introduzione: si presentano gli strumenti dell'analisi, i concetti, densi e vischiosi, di minorité, di minorité religieuse e di majorité. La minorité è la minorité religieuse: laddove la cifra delle relazioni maggioranza/minoranza è la percezione dell'alterità, la religione è promossa a indicatore di questa alterità. Una religione che esiste è una religione che deve essere politicamente organizzata. Strutturare una minoranza religiosa, e appartenervi, diviene il solo mezzo, il solo modo d'esistere in seno a una religione maggioritaria considerata come »dangereuse, dans l'erreur et expansionniste «. Il carattere religioso diventa sui generis e la religione minoritaria "une appartenance identitaire collective, un rempart contre le danger de fonte dans la majorité, une marque d'altérité à ne pas perdre, à cultiver «. Akgönül parla di memoria, quella stessa che mentre per le minoranze religiose autoctone è » une des principales armes de la préservation identitaire «, per le minoranze religiose trapiantate diventa oggetto delle insidie del tempo, costretta a una »reconstruction per- mamente «. Quindi affronta il discorso del dialogo religioso, "fait nouveau « e sospetto cui la minoranza non può che resistere, e del pluralismo che lo sottende. I spegni di fedeltà ‘, presentati dalla maggioranza come procedure di integrazione, assimilazione e inclusione, inclinano il vero senso del pluralismo, mentre il dialogo, limitato a una conoscenza di facciata dell'>altro‘, provoca delle asimmetrie che producono la $>$ resistenza< delle minoranze. Sintomatico, nell'analisi di Akgönül, il dialogo cristianità/islam.

Nel secondo capitolo l'osservazione si sposta al fenomeno minoritario in Turchia per verificare quale sia stato il peso delle minoranze nonmusulmane (greci-ortodossi, armeni-gregoriani ed ebrei) nel processo, tardivo rispetto al resto d'Europa, di costruzione dell'identità turca. Akgönül individua tre momenti fondanti: l'ottomanesimo, l'alternativa alla cristianità e la nazione turca. L'ottomanesimo, in particolare, rappresenta la fusione del sistema sociale ottomano e il resultato del processo di annientamento dei particolarismi etno-religiosi delle millet; esso è la premessa per formare una nazione basata sul sentimento d'appartenenza individuale all'ottomanité e per creare »une altérité islamica - face aux nations occidentales «. La turcité, invece, reinventata dai marcatori identitari e glorificata da un passato tutto turco, è costruita paradossalmente e allo stesso tempo contro l'imperialismo occidentale e sul modello occidentale. In questo gioco di contrasti e acquisizioni, lo stesso status di minoranza è un modello preso in prestito dall'esperienza occidentale. Questo grazie anche al ruolo pedagogico dei trattati internazionali che, a partire dal XIX secolo, hanno imposto la terminologia dell'Occidente e sollecitato l'Oriente ottomano a una vera e propria "intériorisation du vocabulaire de l'adversaire «. Akgönül cita i trattati di Parigi (I 856) 
e Losanna (I923) per aver, rispettivamente, introdotto de facto la sminoranzar come concetto alternativo alla millet e cancellato l'organizzazione pluralista ottomana delle millet e le sue pericolose conseguenze a favore di un nuovo progetto di nazione.

Il terzo capitolo guarda all'appartenenza religiosa nella sua dimensione transnazionale, con particolare attenzione al caso dei musulmani turchi greci, a quel "petit morceau« cioè di nazione turca che dalla Grecia propone un modello di minoranza religiosa valido per gli altri gruppi minoritari sparsi su tutto il territorio europeo. Nella prospettiva dei rapporti grecoturchi il concetto di nazione è liquido e nasconde in sé una dimensione intimamente transnazionale. Le migrazioni, i cambiamenti di confine frequenti, i déplacements di popolazioni sono infatti tutti gli indicatori della fluidità del concetto di nazione, e contribuiscono a creare de facto, quando non de jure, delle vere e proprie »nations éclatées « tra più stati sovrani. Il risultato finale è la produzione di incroci di coscienze identitarie: Akgönül parla di »double appartenance«, senza fare mistero dei problemi di reciprocità che, per esempio, nel caso dei rapporti tra minoranza turca in Grecia e greca in Turchia, essa porta con sé. Ciò che rimane esclusivo è, invece e ancora una volta, il fenomeno di appartenenza religiosa. Questo vale anche in contesti di pluriappartenenza nazionale, come in quello dei cittadini francesi di origine turca. Il processo di costruzione di un'identità turca, già »symptomatique" di per sé, subisce in condizione di minoranza un'ulteriore complicazione. Per i quasi cinque milioni di turchi »de l'extérieur « che vivono in Europa, infatti, i criteri d'affinamento del sentimento di appartenenza al gruppo sono in piena ricomposizione e necessitano di un continuo sascolto< della comunità originaria. S'in- travede quel meccanismo che Benedict Anderson definiva delle »imagined communities « e l'elemento della »transmission d'une memoire collective « diventa »indispensable .

Nel quarto capitolo Akgönül entra nel dettaglio della questione dell'appartenenza, nella sua duplice dimensione di esclusività religiosa e di pluralità nazionale, con particolare attenzione alla prospettiva dei "Turcs en minorité « in Francia. Lì la turcité, dice Akgönül, resiste e diventa attaccamento al territorio d'origine, trasmissione della lingua turca alle generazioni successive, perpetuarsi di comportamenti della tradizione religiosa islamica. Riprendendo il tema della memoria e delle sue strategie di ricomposizione (prima fra tutte quella della cosiddetta " première génération perpétuelle«), Akgönül parla di legami fisici e intellettuali che i turchi rinnovano continuamente con la madrepatria grazie anche alla mediazione delle tecniche di comunicazione, mentre la nazionalità, intesa come cittadinanza da conservare, come corollario cioè del paradigma moderno dello stato-nazione, ne rinnova il legame giuridico. In ragione di ciò, nei decenni passati i governi turchi hanno scoraggiato con forza l'ottenimento della cittadinanza del paese di residenza, per paura che quei turchi potessero perdere la loro turcité e allontanarsi definitivamente dalla madrepatria. Nel 2002 c'è stato un cambio di rotta: i turchi seuropei<, infatti, sono stati incoraggiati a ottenere la cittadianza del paese di residenza, al fine di poter partecipare attivamente alla vita politica nazionale e locale difendendo al meglio, e da una posizione privilegiata, gli interessi della minoranza. E se, in questo contesto di integrazione`, la lingua turca ha subito un graduale indebolimento generazionale, la religione, conservando la sua esclusività d'appartenenza, si è rivelata il "marqueur principal de la turcité « fuori dalla Turchia, una 
barriera »conjoncturelle « che rende ancora oggi i francesi »de souche « incapaci di includere i concittadini allogeni nell'idea stessa di nazione.

In Turchia, intanto, il processo di costruzione nazionale è diviso attualmente tra una corrente promotrice di una definizione rigida di turcité basata sulla lingua, sulla religione sunnita, sulla cultura turca e sull'attaccamento alle origini centro-asiatiche e ottomane; e una seconda inclusiva che, al contrario, partendo da una premessa etnocentrica, propone un'identità nazionale »de Turquie«, »Türkiyeli«, che inglobi componenti etnico-religiose musulmane (curde e alevite) e minoranze non-musulmane. Se in Turchia, come altrove, resta auspicabile una fase del processo di costructione nazionale che Akgönül definisce di »individualizzazione identitaria e comunitarizzazione sociale «, in Francia

\section{Caveat emptor*}

Der römische Käufer wird vor Arglist des Verkäufers geschützt, er darf sich auf dessen Zusicherungen verlassen sowie auf die Abwesenheit bestimmter nicht erkennbarer Mängel der Kaufsache. Verbleibende Risiken des Geschäfts weist man lakonisch ihm zu: periculum est emptoris - »Die Gefahr trägt der Käufer «. Das Formular für die Versteigerung von Wein in Gärfässern (vinum in doliis), das Cato in seinem Handbuch des Landbaus (de agri cultura; Mitte 2. Jh. v. Chr.) dem römischen Grundbesitzer neben den Verkaufsbedingungen für hängende Oliven und hängenden Wein an die Hand gibt, ist Éva Jakabs Ausgangspunkt bei ihrer (inzwischen preisgekrönten $)^{\mathbf{I}}$ Untersuchung der römischen und griechisch-hellenistischen Vertragsge- la minoranza rimane una normativizzazione e un'autorappresentazione dell'appartenenza dove l'individuo non compare se non come membro di un gruppo marginalizzato. Quando, infatti, il confine maggioranza/minoranza sembra cedere per la richiesta della maggioranza di un graduale adattamento dei riti minoritari al proprio contesto, la minoranza percepisce il cambiamento come imposto e segno di »déculturation «. Akgönül parla di una vera e propria »peur de déculturation « della minoranza, paura che porta sarcasticamente a sottolineare come, ancora oggi, uno degli insulti peggiori che si possa fare a un turco che vive in Francia sia quello di dirgli d'essersi >francesizzato<.

Eliana Augusti

staltung beim Weinkauf, von Leistungspflichten, Mängelhaftung und Gefahrtragung in den Urkunden. Der Weinhandel kennt besondere Risiken: Wein kann umschlagen; ein Fass kann auslaufen.

Jakabs intensive Arbeit mit unterschiedlichsten Quellen eröffnet Diskussionsfelder der juristischen Papyrologie und Epigraphik, der Textkritik, der Archäologie. Sie zeigt eindrücklich, wie willkürlich eine Aussonderung griechischer und nichtjuristischer Quellen wäre; wie fatal es wäre, der eigenen minutiösen Arbeit am Text Grenzen zu setzen, um schneller zur »großen These " zu gelangen. Auf dieser Ebene der präzisen Quellenarbeit muss die kritische Auseinandersetzung mit Jakabs Buch stattfinden.

\footnotetext{
Éva JaKab, Risikomanagement beim Weinkauf. Periculum und Praxis im Imperium Romanum

I Preis des Deutschen Rechtshistorikertages $20 \mathrm{IO}$. rusforschung und Antiken Rechtsgeschichte, 99. Heft) München: C. H. Beck 2009 , 284 S., ISBN 978-3-406-58285-I
} 